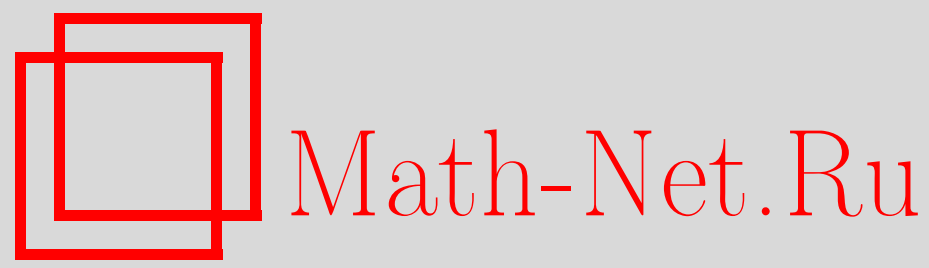

Л. З. Фишман, О сохранении состояний равновесия и их устойчивости при замене непрерывной системы дискретной, построенной по методу Рунге-Кутта, Матем. заметки, 1996, том 59, выпуск $5,784-787$

DOI: https://doi.org/10.4213/mzm1776

Использование Общероссийского математического портала MathNet.Ru подразумевает, что вы прочитали и согласны с пользовательским соглашением

http://www . mathnet.ru/rus/agreement

Параметры загрузки:

IP : 52.23 .180 .231

26 апреля 2023 г., 15:54:48

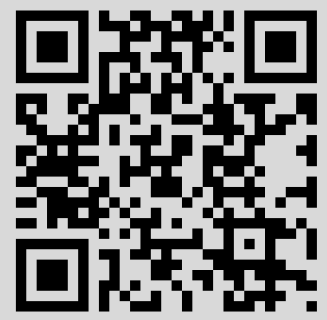




\section{О СОХРАНЕНИИ СОСТОЯНИЙ РАВНОВЕСИЯ И ИХ УСТОЙЧИВОСТИ ПРИ ЗАМЕНЕ НЕПРЕРЫВНОЙ СИСТЕМЫ ДИСКРЕТНОЙ, ПОСТРОЕННОЙ ПО МЕТОДУ РУНГЕ-КУТТА}

\section{Л. З. Фишман}

Рассматривается непрерьвная система второго порядка

$$
\dot{x}=F(x),
$$

где $F(x)$ - аналитическая вектор-функция.

Пусть система (1) заменяется дискретной системой, построенной по явному $s$-этапному методу Рунге-Кутта $p$-го порядка

где

$$
\bar{x}=x+h \sum_{i=1}^{s} b_{i} F\left(Y_{i}\right)
$$

$$
Y_{1}=x, \quad Y_{i}=x+h \sum_{j=1}^{s} c_{i j} F\left(Y_{j}\right), \quad 2 \leqslant i \leqslant s,
$$

$c_{i j}=0, i \leqslant j, s \geqslant p \geqslant 1, \sum_{i=1}^{s} b_{i}=1, h>0$.

При замене дифференциальных уравнений дискретными, построенными по методам Эйлера и Рунге-Кутта, возникает [1]-[6] вопрос о сохранении состояний равновесия дифференциальных уравнений и их характера. Как известно [2], [3], все состояния равновесия дифференциальых уравнений являются также и состояниями равновесия соответствующих дискретных уравнений. Дискретные уравнения, построенные для скалярных дифференциальных уравнений по методам Рунге-Кутта порядка $p>1$ могут иметь состояния равновесия, которых нет у исходных диффференциальных уравнений. Кроме того, у них могут быть периодические точки периода большего, чем единица, которых нет у исходных дифференциальных уравнений. Такие состояния равновесия и периодические точки названы ложными. В случае, когда дискретные уравнения построены по методу Эйлера, у них не может быть ложных состояний равновесия, но могут быть ложные периодические точки периода болшшего, чем единица.

Сохранение характера границ областей устойчивости состояний равновесия типа фокус и узел при замене системы (1) системой (2), построенной по методу Эйлера, показано в [7]. В [8]-[11] даны условия сохранения характера границы области устойчивости фокуса системы (1) при замене ее произвольными системами (2). Показана возможность несохранения характера границы области устойчивости фокуса системы (1).

В данной работе обобщаются и развиваются резултаты [7]-[11]. Даются условия (теорема 1) отсутствия у системы (2) ложных состояний равновесия и периодических точек периода большего, чем единица, при достаточно малых шагах $h$. Показано, что при невьполнении условия достаточной малости $h$, системы $(2)$, построенные по 2- и 4-этапньм явным методам Рунге-Кутта 2-го и 4-го порядка, могут иметь ложные состояния равновесия. Даются условия (теорема 2) сохранения характера границы области устойчивости состояния равновесия типа узел системы (1) при замене ее произвольными системами (2).

Работа выполнена при поддержке программы "Университеты России", направление "Фундаментальные проблемы математики и механики", грант № 1.5.21. 
1. Сохранение состояний равновесия системы (1) при замене ее системой (2). Все состояния равновесия системы (1) являются состояниями равновесия системы (2). Возникает вопрос: может ли система (2) иметь ложные состояния равновесия и периодические точки периода большего, чем единица. В случае, когда (2) построена по методу Эйлера, у нее не может быть ложных состояний равновесия, но могут быть ложные периодические точки периода большего, чем единица. В случае, когда система (2) построена по явному методу Рунге-Кутта любого порядка, достаточные условия отсутствия ложных состояний равновесия и периодических точек периода большего, чем единица, дает следующая

ТЕОрема 1. Пусть система (1) имеет грубые состояния равновесия $\mathrm{O}_{1}, \mathrm{O}_{2}, \ldots, \mathrm{O}_{n}$, расположеннье внутри замкнутой ограниченной области $G$ плоскости $\left(x_{1}, x_{2}\right)$. Пусть гранича $G$ является простой замкнутой кривой. Тогда существует такое достаточно малое $h_{1}$, что при $0<h \leqslant h_{1}$ система (2) имеет в области $G$ только состояния равновесия $\mathrm{O}_{1}, \mathrm{O}_{2}, \ldots, \mathrm{O}_{n}$ u не имеет других состояний равновесия и периодических точек периода большего, чем единича.

ДокАЗАТЕЛЬСТво теоремы следует из того, что уравнение для координат состояний равновесия и периодических точек периода $2,3, \ldots, N$, где $N$ не зависит от $h$, системы (2) приводится к виду

$$
F(x)+h \Omega(x, h)=0,
$$

где $\Omega(x, h)$ - аналитическая по $(x, h)$ функция.

Рассмотрим систему

$$
\dot{x}_{1}=-x_{1}+x_{1}^{3}-x_{1} x_{2}^{2}, \quad \dot{x}_{2}=x_{2}+x_{1}^{2},
$$

представляющую собой частньй случай системы (1).

Покажем, что системы (2), построенные для (3) по 2-этапному явному методу Рунге-Кутта 2-го порядка (РК2) и по классическому методу Рунге-Кутта 4-го порядка (РК4) при некоторых значениях шага $h$ имеют состояния равновесия, которых нет у системы (3).

В окрестности состояния равновесия $x_{1}=x_{2}=0$ системы PK2 и РК4 записываются в виде

$$
\begin{aligned}
& \bar{x}_{1}=\lambda_{1}(h) x_{1}+h c_{1}(h) x_{1}^{3}+h \Omega_{1}\left(x_{1}, x_{2}, h\right), \\
& \bar{x}_{2}=\lambda_{2}(h) x_{2}+h \Omega_{2}\left(x_{1}, x_{2}, h\right),
\end{aligned}
$$

где $\Omega_{1}\left(x_{1}, x_{2}, h\right)$ и $\Omega_{2}\left(x_{1}, x_{2}, h\right)$ начинаются с членов не ниже 3 -го и 2-го порядка по $\left(x_{1}, x_{2}\right)$.

Корень $\lambda_{1}(h)$ удовлетворяет неравенству $\left|\lambda_{1}(h)\right|<1$ при $0<h \leqslant h^{*}$ и $\left|\lambda_{1}(h)\right|>1$ при $h>h^{*}, \lambda_{1}\left(h^{*}\right)=1$, где $\left(-h^{*}, 0\right)$ - интервал абсолютной устойчивости $\left[12\right.$, с. 47] соответствующего метода Рунге-Кутта. Корень $\lambda_{2}(h)$ удовлетворяет неравенству $\lambda_{2}(h)>1$ при $h>0$. Для коэффициента выполнено $c_{1}\left(h^{*}\right)<0$.

При увеличении $h$ от $h=h^{*}$ имеет место бифуркация рождения их трехкратного состояния равновесия $x_{1}=x_{2}=0$ системы (4) двух ложных грубых состояний равновесия, которых нет у системы (3). Отметим, что для систем PK2 и PK4 величины $h^{*}$ равны 2 и 2.785.

Таким образом, при дискретизации системы (1) по явному 2-этапному методу Рунге-Кутта 2-го порядка и по классическому методу Рунге-Кутта 4-го порядка могут появляться ложные состояния равновесия. 
2. Сохранение характера границы области устойчивости состояния равновесия типа узел при замене системы (1) системой (2). Пусть $F(x)=A x+f(x)$, матрица $A$ равна $\left[a_{i k}\right], i, k=1,2, f(0)=0$ и в окрестности точки $x=0$ компоненты вектор-функции $f\left(x_{1}, x_{2}\right)$ разлагаются в ряды по степеням $\left(x_{1}, x_{2}\right)$ не ниже второй. Пусть $r=-\left(a_{11}+a_{22}\right), q=a_{11} a_{22}-a_{12} a_{21}$ и $r^{2}-4 q>0$.

Полупрямая $r>0, q=0$ на плоскости параметров $(r, q)$ является границей $\left(\Gamma_{1}\right)$ области устойчивости узла $x=0$ системы (1). Система (2) при любом $h$ также имеет состояние равновесия $x=0$. При замене системы (1) системой (2) граница $\Gamma_{1}$ системы (1) переходит в границу $0<r<r^{*} / h, q=0\left(\Gamma_{2}\right)$ области устойчивости состояния равновесия $x=0$ системы $(2)$, где $\left(-r^{*}, 0\right)$ - интервал абсолютной устойчивости метода Рунге-Кутта, по которому построена система (2). На границе $\Gamma_{2}$ состояние равновесия $x=0$ системы (2) имеет корни $\lambda_{1}=1$ и $\lambda_{2}$, удовлетворяющий неравенству $\left|\lambda_{2}\right|<1$.

Пусть $l_{1}$ и $l_{2}$ - первая и вторая ляпуновские величины системы $(1), g_{1}$ и $g_{2}$ - величины, аналогичные первой и второй ляпуновским, системы $(2)$, найденные в точке $q=0, r=r_{0}$ границ $\Gamma_{1}$ и $\Gamma_{2}$.

Имеет место следующая

Tеорема 2. Пусть $q=0, r=r_{0}, 0<h<r^{*} / r_{0}$. Тогда если $l_{1} \neq 0$, mо $g_{1}=h l_{1}, u$ состояние равновесия $x=0$ системы (2) так жсе, как $u$ системы (1), - двукратное, неустойчивое. Если $l_{1}=0, l_{2}<0\left(l_{2}>0\right)$, то $g_{2}=h l_{2}$, и состояние равновесия $x=0$ системы (2) так же, как (1), трехкратное, устойчивое (неустойчивое).

ДоКАЗАТЕЛЬСтво. Системы (1) и (2) при $q=0, r=r_{0}$ с помощью замены переменных приводятся к виду

$$
\begin{aligned}
& \dot{x}_{k}=p_{k} x_{k}+\sum_{n \geqslant 2} \sum_{r+q=n} a_{r q}^{(k)} x_{1}^{r} x_{2}^{q}, \\
& \bar{x}_{k}=\lambda_{k} x_{k}+h \sum_{n \geqslant 2} \sum_{r+q=n} b_{r q}^{(k)} x_{1}^{r} x_{2}^{q},
\end{aligned}
$$

где $p_{1}=0, p_{2}=-r_{0}, \lambda_{1}=1,\left|\lambda_{2}\right|<1$, коэффициенты $b_{r q}^{(k)}$ выражаются через коэффициенты $a_{r q}^{(k)}$, причем $b_{20}^{(1)}=a_{20}^{(1)}, k=1,2$.

Так как величина $g_{1}$, аналогичная первой ляпуновской для системы (6), равна $h b_{20}^{(1)}$, первая ляпуновская величина $l_{1}$ системы (5) равна $a_{20}^{(1)}$ и $b_{20}^{(1)}=a_{20}^{(1)}$, то получаем равенство $g_{1}=h l_{1}$, которое и требовалось доказать. Из [13], [14] следует, что при условии $a_{20}^{(1)}=l_{1}=g_{1}=0$ вторая ляпуновская величина $l_{2}$ для системы (5) равна $a_{30}^{(1)}+a_{11}^{(1)} a_{20}^{(2)} / r_{0}$, а величина $g_{2}$, аналогичная второй ляпуновской для системы (6), равна $h\left[b_{30}^{(1)}+h b_{11}^{(1)} b_{20}^{(2)} /\left(1-\lambda_{2}\right)\right]$. Подставляя в формулу для $g_{2}$ выражения для коэффициентов $b_{30}^{(1)}, b_{11}^{(1)}, b_{20}^{(2)}$ через $a_{20}^{(1)}, a_{11}^{(1)}, a_{20}^{(2)}, a_{30}^{(1)}$, придем к равенству $g_{2}=h l_{2}$, которое и требовалось доказать.

СлЕДСТВИЕ. При выполнении условий, сформулированных в теореме 2, имеет место сохранение характера әраницы $\Gamma_{1}$ системы (1) в точке $q=0$, $r=r_{0}$ при замене (1) системой (2). 


\section{СПИСОК ЦИТИРОВАННОЙ ЛИТЕРАТУРЫ}

1. Beyn W.-J. // SIAM J. Numer. Anal. 1987. V. 24. № 5. P. 1095-1113. 2. Iserles A. // IMA J. Numer. Anal. 1990. V. 10. №1. P. 3-9. 3. Hairer E., Iserles A., Sanz-Serna J. M. // Numer. Math. 1990. V. 58. P. 243-254. 4. Iserles A., Peplov A. T., Stuart A. M. // SIAM J. Numer. Anal. 1991. V. 28. №6. P. 1723-1751. 5. Yee H. C., Sweby P. H., Griffits D. F. // J. Comput. Phys. 1991. V. 97. P. 249-310. 6. Cartwright J., Piro O. // Int. J. of Bifurcation and Chaos. 1992. V. 2. № 3. Р. 427-449. 7. Белых В. Н., Сенниковский Я. Н. О качественном исследовании отображений с помощью систем дифференциальных уравнений // Деп. ВИНИТИ. № 5042-В86. 8. Фишман Л. З. // Автоматика и телемеханика. 1991. № 4. С. 186-189. 9. Фишман Л. З. // Докл. РАН. 1992. Т. 327. № 1. С. 32-36. 10. Фишман Л. З. // Динамика систем. Динамика и оптимизация / ред. Ю.И. Неймарк. Межвуз. тематич. сб. научн. тр. Н. Новгород: НГУ, 1992. С. 124-136. 11. Фишман Л. З. // Матем. заметки. 1992. Т. 52. № 6. С. 131-139. 12. Дж. Холл, Дж. Уатт (ред. ) Современные численные методы решения обыкновенных дифференциальных уравнений. М.: Мир, 1979. 13. Баутин Н. Н. Поведение динамических систем вблизи границ области устойчивости. М.: Наука, 1984. 14. Неймарк Ю. И. // Изв. вузов. Радиофизика. 1958. Т. 1. № 2. С. 95-130. 